\title{
ENFERMEDAD INFLAMATORIA INTESTINAL ASOCIADA A LINFOMA NO HODGKIN EN PACIENTE CON VIH
}

\author{
INFLAMMATORY BOWEL DISEASE ASSOCIATED WITH \\ NON HODGKIN LYMPHOMA IN AN HIV PATIENT. $X$ \\ DOENÇA INFLAMATÓRIA INTESTINAL ASSOCIADA A \\ LINFOMA NÃO-HODGKIN EM PACIENTES COM HIV
}

\section{JAMES NEIRA BORJA', LÚVER MACÍAS JARA', JHON CORONEL RUILOVA', DIEGO PAREDES CALAHORRANO', FÁtIMA NEIRA VERDUGA ${ }^{2}$}

\author{
1 Hospital Luis Vernaza, Guayaquil, Ecuador \\ 2 OmniHospital, Guayaquil, Ecuador
}

Resumen

Introducción: las enfermedades inflamatorias intestinales tienen una incidencia de 3,7-7,3 casos por 100.000 habitantes. Un estudio presentado en el año 2008 las personas portadoras del virus de inmunodeficiencia humana tendrían el doble de posibilidades de sufrir colitis ulcerosa. El linfoma no Hodgkin es la segunda neoplasia en pacientes con enfermedad HIV/sida, existen reportes sobre la asociación de linfoma no Hodgkin con enfermedad inflamatoria intestinal. Objetivo: dar a conocer que la enfermedad inflamatoria Intestinal puede estar asociado en los pacientes con linfoma no Hodgkin con VIH/SIDA. Descripción del caso: paciente de 52 años de edad que presenta un mes de evolución con dolor en hemiabdomen izquierdo acompañado de constipación, junto con fases de estreñimiento alternado con diarreas de consistencia mucosa que generaron pérdida de peso. Discusión: el linfoma no Hodgkin y el linfoma Hodgkin están asociados a la infección por VIH en adultos; el linfoma asociado a VIH puede ser una enfermedad definitoria de SIDA o aparecer en la evolución del SIDA. Conclusión: a nivel mundial pocos casos se han reportado acerca de esta coexistencia entre infección por el virus de inmunodeficiencia humana en pacientes con enfermedad inflamatoria intestinal y los efectos de una enfermedad sobre la otra. PALABRAS CLAVE: enfermedad inflamatoria intestinal, linfoma no Hodgkin, síndrome de inmunodeficiencia adquirida.

Abstract

Introduction: the incidence of Inflammatory bowel disease is 3.7-7.3 cases per capita. In a study published in 2008, HIV patients show twice the odds to develop ulcerative colitis. Non-Hodgkin lymphoma is the second most common neoplasia in HIV and AIDS patients. There are reports of an association between non-Hodgkin Lymphoma and Inflammatory bowel disease. Objective: to demonstrate a possible association of Inflammatory Bowel disease and non-Hodgkin Lymphoma in patients with HIV and AIDS. Case presentation: a 52-year-old patient is admitted with left abdominal pain of 1 month of evolution accompanied with constipation along with mucous diarrhea that caused weight loss. Discussion:non-Hodgkin lymphoma and Hodgkin Lymphoma are associated with HIV infection in adults. These pathologies can be and AIDS-defining illnesses or a part of AIDS's evolution. Conclusion: there are few reported cases of the impact and association between AIDS and Inflammatory bowel disease.

KEYWORDS: inflamation; lymphoma non-Hodgkin's; acquired inunodeficiency syndrome.

Resumo

INTRODUÇão: as doenças inflamatórias intestinais têm uma incidência de 3,7 a 7,3 casos por 100.000 habitantes, um estudo apresentado em 2008 as pessoas com HIV têm o dobro da probabilidade de desenvolver colite ulcerosa. El linfoma não-Hodgkin é o segundo neoplasia em pacientes com HIV / AIDS, há relatos de associação de linfoma não-Hodgkin com doença inflamatória intestinal. Objetivo: dar a conhecer que a doença inflamatória intestinal pode estar associada em pacientes com linfoma não-Hodgkin com HIV / AIDS. Relato de caso: paciente de 52 anos de idade que apresenta doença de um mês da evolução caraterizado dor abdominal acompanhada por constipação, juntamente com fases obstipação alternando com consistência diarreia mucosa que o fizeram perder peso. Discussão: o LNH e o linfoma de Hodgkin (LH) estão associados à infecção por HIV em adultos e o linfoma associado ao HIV pode ser uma doença definidora da AIDS ou aparecer na evolução da AIDS. Conclusão: em todo o mundo, poucos casos foram relatados sobre essa coexistência entre a infecção pelo vírus da imunodeficiência humana em pacientes com doença inflamatória intestinal e os efeitos de uma doença no outro.

PALABRAS-CHAVE: inflamação intestinais, linfoma não-Hodgkin, síndrome da imunodeficiência adquirida. 
INTRODUCCIÓN

Las enfermedades inflamatorias Intestinales (EII) incluye a la colitis ulcerosa (CU), enfermedad de Crohn (EC) y colitis indeterminada $(\mathrm{CI})^{1}$; la etiología es desconocida, se reconoce que factores genéticos, inmunológicos y ambientales tienen un papel importante en la patogénesis de esta enfermedad: presenta una incidencia de 3,7-7,3 casos por 100.000 habitantes, siendo muy variable según la población que se estudie. ${ }^{2}$ Los síntomas iniciales de estas dos entidades pueden ser: diarrea sanguinolenta así como dolor abdominal difuso, pérdida de peso y malestar general. ${ }^{3}$

Según un estudio presentado en el año 2008, las personas con VIH tendrían el doble de posibilidades de sufrir colitis ulcerosa que la población general, aunque no se ha encontrado una relación directa con el recuento de CD4 en el momento del diagnóstico de enfermedad inflamatoria intestinal. ${ }^{4}$

El linfoma no Hodgkin(LNH) constituye un grupo heterogéneo de neoplasias malignas linfoproliferativas; se origina en los tejidos linfoides, su pronóstico depende del tipo histológico, el estadio y el tratamiento; son más frecuentes en adultos a partir de los 50 años y es la segunda neoplasia en pacientes con enfermedad HIV/sida, luego del sarcoma de Kaposi. Existen pocos reportes sobre la asociación de linfoma no Hodgkin con enfermedad inflamatoria intestinal, aunque diversos estudios sugieren que la incidencia se incrementa con el aumento del uso de la terapia inmunosupresora.

El objetivo es dar a conocer el presente caso clínico en el que la enfermedad inflamatoria intestinal puede estar asociada en los pacientes con linfoma no Hodgkin con VIH/SIDA.

REPORTE DE CASO

Paciente masculino de 52 años de edad sin antecedentes patológicos, con hábitos de fumador crónico y alcohólico crónico, acude por presentar cuadro clínico de más de un mes de evolución caracterizado por dolor sin causa aparente, localizado en hemiabdomen izquierdo acompañado de constipación, junto con fases de estreñimiento alternado con diarreas de consistencia mucosa que generó pérdida de peso; además, anorexia, astenia, malestar general y dispepsias. Al exámen físico se evidencia una gran masa abdominal de consistencia dura, no reductible, con bordes regulares a nivel de hemiabdomen izquierdo (Figura 1).

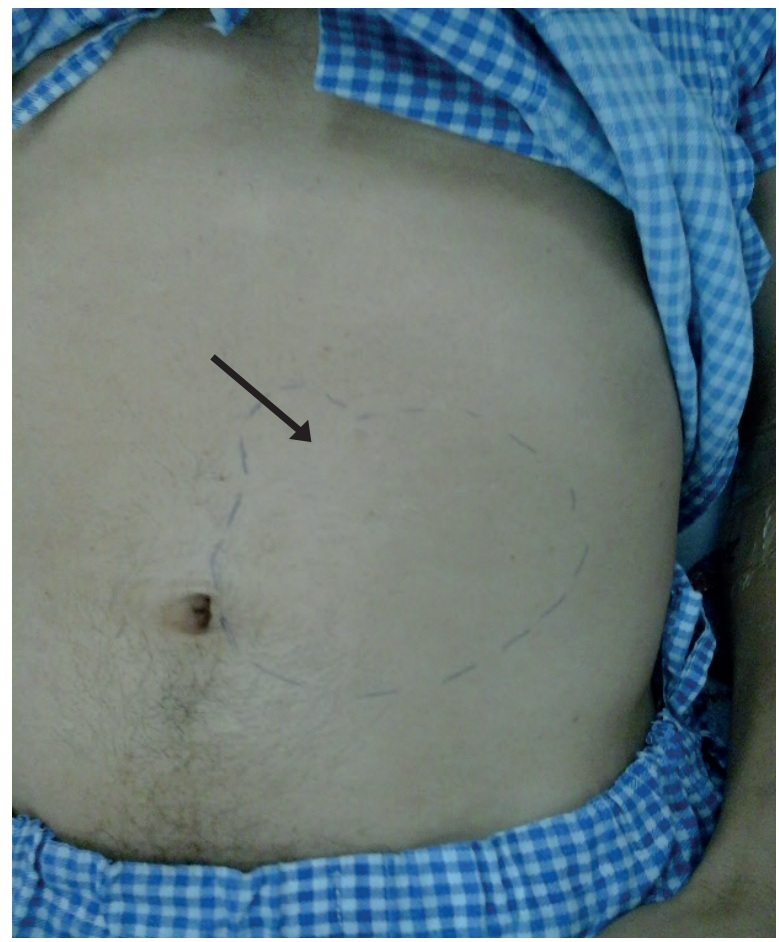

Figura 1. muestra una masa abdominal en flanco izquierdo, (flecha).

Dentro de los resultados de interés en los exámenes especiales indicados se observa: beta $2 \mathrm{mi}^{-}$ croglobulina $32 \mathrm{ug} / \mathrm{mL}$, anti chagas no reactivo, anti HAV IgC reactivo, anti HBcIgM no reactivo, anti $\mathrm{HBc}$ reactivo, anti $\mathrm{HBs} 78.75$, hepatitis $\mathrm{A}, \mathrm{B}$ y C negativos, CA 19-9 12,71 u/mL, CA $12515,26 \mathrm{u} /$ mL, CD4 516 células/uL con CD3 y CD8 normales, citomegalovirus IgC 182,5 AU/mL, Epstein Barr IgG positivo; Monotest negativo; toxoplasmosis IgG 200 IU/mL; VIH reactivo y VDRL no reactivo. El resultado del Western Blot HIV, positivo. Carga viral para HIV-1: 51236, se recomendó test de resistencia Genotipo VIH (mínimo <34 copias de ARN-HIV/mL, máximo >1000000).

En la hemoquímica se detectaron valores hematocrito $29.5 \%$, eosinófilos $0,86 \times 10 / \mathrm{uL}$, eosinófilos $15,1 \%$, neutrófilos $42,3 \%$, HCM 27,0 pg, CHCM $32,6 \mathrm{~g} / \mathrm{dL}$, colesterol HDL $26 \mathrm{mg} / \mathrm{dL}$, hierro $37 \mathrm{ug} /$ $\mathrm{dL}$, transferrina $183 \mathrm{mg} / \mathrm{dL}$, PCR 33,81 mg/dL, prealbúmina $12 \mathrm{mg} / \mathrm{dL}$, sero-albúmina $3,47 \mathrm{~g} / \mathrm{dL}$ y sero-globulina $3,72 \mathrm{~g} / \mathrm{dL}$.

En los estudios imagenológicos se reporta TAC de abdomen esplenomegalia más masa de contornos irregulares con áreas de necrosis en su interior retroperitoneal que no compromete al colon descendente en el hemiabdomen izquierdo (Figura 2). 


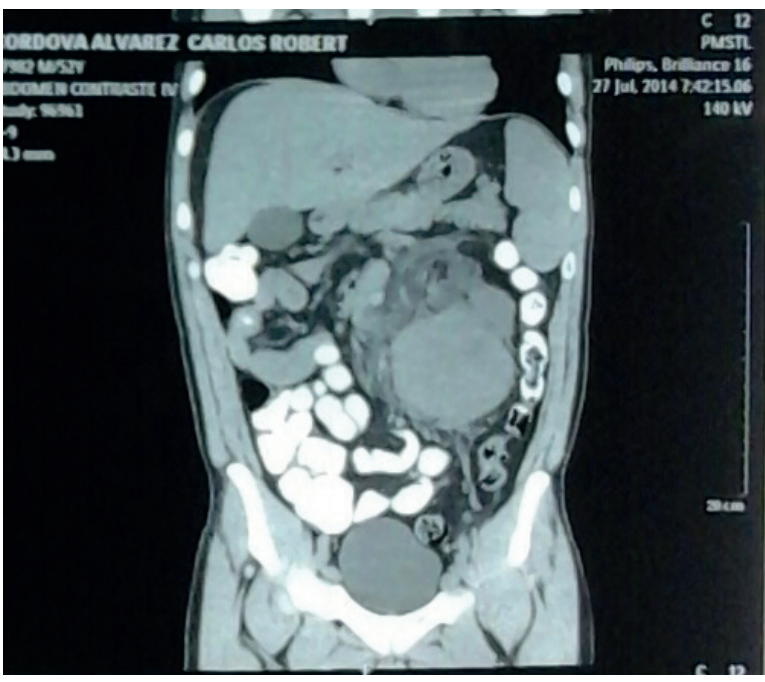

Figura 2. TAC de abdomen corte coronal. Se observa una masa de contornos irregulares (flecha) con áreas de necrosis en su interior retroperitoneal que no compromete al colon descendente en el hemiabdomen izquierdo.

Posteriormente se realizó VEDA cuya impresión diagnóstica fue gastropatía congestiva antral moderada. Al mismo tiempo se efectuó video colonoscopía que describió rectorragia más 2 úlceras rectales compatibles con proctitis inflamatoria inespecífica (Figura 3).

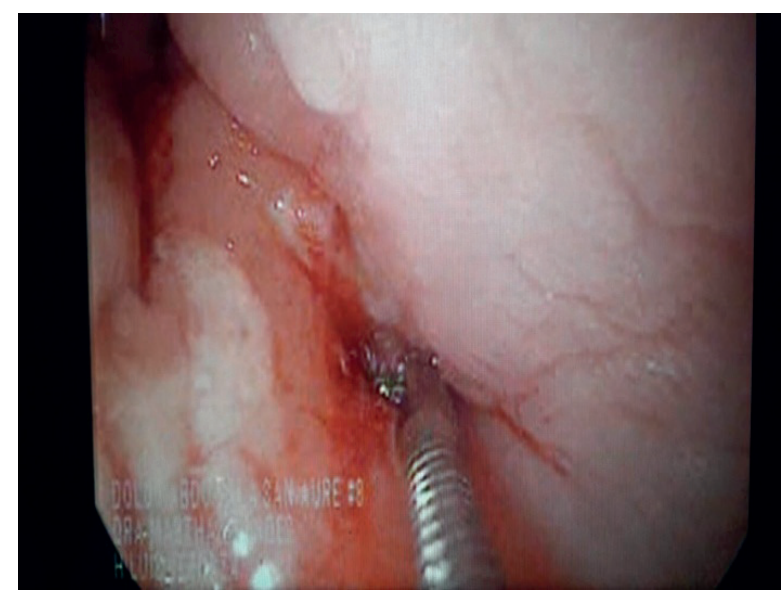

Figura 3. colonoscopía describiendo rectorragia más lesiones ulcerosas rectales compatibles con proctitis inflamatoria inespecífica.

En ambos exámenes se tomaron biopsias cuyos resultados se describen a continuación:

Muestra de estómago (antro y cuerpo): examen microscópico: mucosa gástrica de antro y cuerpo con glándulas conservadas. La lámina propia muestra un moderado infiltrado de linfocitos, células plasmáticas, formación de folículos linfoides y abundantes polimorfo nucleares. Las criptas son de tamaño irregular. En el epitelio hay cambios reactivos.
Impresión diagnóstica: gastritis crónica severa. hiperplasia linfoide. Colonización moderada de H. pylori.

Muestra de recto: examen microscópico: mucosa de recto presenta edema de la lámina propia, infiltrado ligero de linfocitos, células plasmáticas; las células son de tamaño uniforme, epitelio con ligeros cambios reactivos.

Impresión diagnóstica: colitis crónica ligera inespecífica.

Después de 48 horas de haberse realizado estudio endoscópico, se procede a realizar biopsia de la masa tumoral dirigida por tomografía.

Examen microscópico: proliferación neoplásica maligna difusa constituida por células grandes de amplio citoplasma claro, núcleo vesiculoso, nucléolo prominente, abundantes mitosis, necrosis focal.

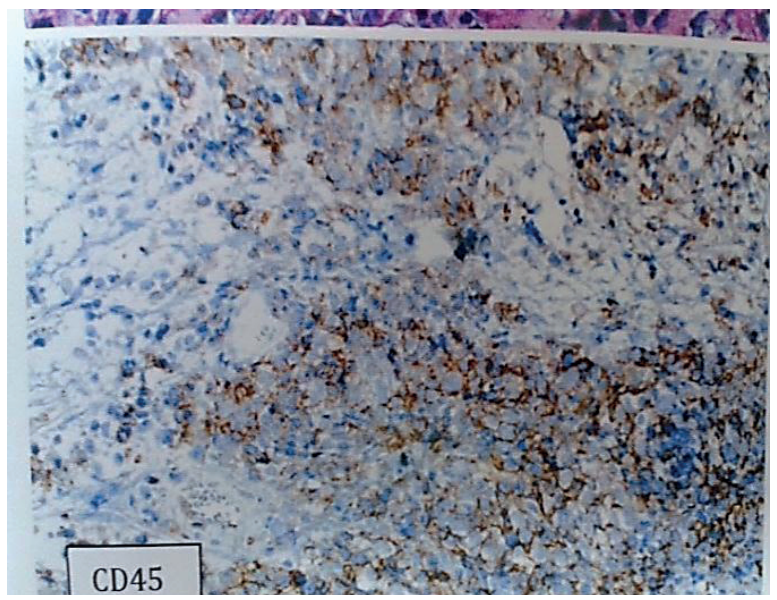

Figura 4. Inmunohistoquímica: CD45 positivo. Citoqueratina 8-18 negativa (descarta metástasis de carcinoma). S100 negativa (descarta melanoma).

Diagnóstico: linfoma no Hodgkin difuso de células grandes.

DISCUSIÓN

El LNH y el linfoma Hodgkin (LH) están asociados a la infección por VIH en adultos desde los inicios de la epidemia. El linfoma asociado a VIH puede ser una enfermedad definitoria de SIDA o aparecer en la evolución del mismo. Casi siempre tiene un origen celular B y corresponde principalmente a un linfoma B de células grandes. Se ha señalado que este linfoma aparece como consecuencia de la estimulación causada por el VIH con reactivación de una infección latente por virus Epstein Barr (VEB). ${ }^{10}$ Se presenta con mayor frecuencia en 
pacientes seropositivos al virus de la inmunodeficiencia humana (VIH).Esto es importante porque ocupa el segundo lugar en frecuencia entre las neoplasias vinculadas. La incidencia de $\mathrm{LNH}$ en pacientes con VIH es más de 100 veces la encontrada en la población general. Con la introducción del tratamiento antirretrovírico pareció aumentar el número de pacientes con linfomas asociados al VIH.

El LNH en los pacientes infectados por VIH persiste en nuestro medio como una enfermedad de pésimo pronóstico, con frecuente progresión y recaídas y, adicionalmente, muertes provocadas por aplasia medular post quimioterapia y sus complicaciones infecciosas. Actualmente la mayoría de los pacientes con linfoma e infección por VIH logran una curación de la enfermedad con sobrevida global a 5 años mayor de $60 \%$, situación que no se observa en la casuística nacional.

La incorporación de rituximab y la aplicación de esquemas de quimioterapia ajustados a la histología y factores de riesgo en el contexto de una mejoría general de la atención de salud de los pacientes del sistema público deberían, en el futuro, mejorar los indicadores de salud. ${ }^{11}$ Todos los datos indican que el incremento de la incidencia es más importante en las personas de edad avanzada, sobre todo en lo que se refiere al linfoma B de células grandes difuso (LBCGD).

Entre los decenios de 1940 y 1980 la incidencia ha aumentado del $4 \%$ anual, lo que representa un incremento del $150 \%$ en este período. Este crecimiento sólo se debe en parte al envejecimiento natural del ser humano. Las razones para argumentar tal crecimiento no están del todo claras, no obstante se han implicado diversos factores etiológicos, que se expresan en procesos de inmunodeficiencia primarios y secundarios; lo representativo y peculiar de esto es el punto en común que comparten todas estas posibles etiologías que es la inmunosupresión como factor de riesgo más claramente definido.

En base a este punto de encuentro se puede reafirmar que los linfomas aparecen en un contexto de supresión y estimulación inmunitarias, que predisponen a una incidencia elevada de la enfermedad, por lo que se ha prestado una atención considerablemente mayor a los cuadros de supresión inmunitaria.

\section{CONCLUSIÓN}

A nivel mundial pocos casos se han reportado acerca de esta coexistencia entre infección por el virus de inmunodeficiencia humana en pacientes con enfermedad inflamatoria intestinal y los efectos de una enfermedad sobre la otra. Menos aún se ha informado casos de pacientes que cursen con estas patologías anexándose la presencia de linfoma no Hodgkin. En base a la participación del sistema inmunitario en la patogénesis de la EII, se podría considerar algún efecto de la infección por VIH/SIDA sobre la EII promoviendo la depleción de los linfocitos CD4 como ocurre en estos pacientes. El VIH afecta el tracto gastrointestinal a través de infecciones oportunistas (cryptosporidium, MAC, etc.) o enfermedades neoplásicas (linfomas, sarcoma de Kapossi). ${ }^{6}$ El efecto de la infección por VIH y SIDA sobre la enfermedad inflamatoria intestinal, específicamente, colitis ulcerativa y enfermedad de Crohn, es controversial. En 1988, en la revista Gastroenterology, el Dr. James SP. reportó un paciente con enfermedad de Crohn de larga evolución que remitió después de haberse infectado con el virus del VIH y a medida que disminuyó la población de linfocitos CD4, sugiriendo un efecto favorable del VIH en cuanto a la actividad de la EII al disminuir el conteo de estas células. ${ }^{6}$ Por otro lado, existen algunos reportes de novo, de colitis ulcerativa y enfermedad de Crohn en pacientes con infección por VIH o SIDA que sugieren lo contrario. ${ }^{8}$ En la actualidad se han realizado estudios indicando que la incidencia de linfoma no Hodgkin se eleva a medida que aumenta el uso de la terapia inmunosupresora. Aunque la EII subyacente puede ser un factor causal en el desarrollo del LNH intestinal, la experiencia adquirida sugiere que los fármacos inmunosupresores pueden aumentar significativamente el riesgo de LNH en la EII. Esto debe compararse con la mejor calidad de vida y la terapia inmunosupresora beneficio clínico previo de los pacientes con EII. ${ }^{7}$ Entre los fármacos que se asocian con este aumento se citan el uso de tiopurinas (azatioprina y 6-mercaptopurina) y de agentes anti-TNF $\alpha .{ }^{9}$

\section{REFERENCIAS BIBLIOGRÁFICAS}

1. Russel MG, Stockbrugger RW. Epidemiology of Inflammatory Bowel Disease: an update. Scand J Gastroenterol 1996; 31: 417-27.

2. Loftus EV Jr. Clinical epidemiology of inflammatory bowel disease: Incidence, prevalence, and environmental influences. Gastroenterology 2004; 126: 1504-17. 
3. Hellers G. Crohn's disease in Stockholm county, 1955-1974: a study of epidemiology, result of surgical treatment and long term prognosis. ActaChirScandSuppl 1979; 490: 1-84.

4. Landy J, Gazzard B, Harbord M. Inflammatory Bowel Disease in HIV Seropositive Individuals: Analysis of a Large Cohort. Digestive Disease Week (DDW) 2008. San Diego, CA. May 17-22, 2008. Abstract T1193

5. Coexistencia de sida y proctitis ulcerativa en un paciente colombiano caso clínico Interinstitucional Yezid Alfonso Farfán Q. MD, Antonio José Restrepo MD, Julián David Martínez MD, Mario Humberto Rey MD, Juan Carlos Marulanda MD, Juan Carlos Molano MD, Martín Garzón O. MD. Rev Col Gas troenterol / 21 (2) 2006, pag. 127

6. Aumento de la incidencia de linfoma no Hodgkin en pacientes con enfermedad inflamatoria intestinal en tratamiento inmunosupresor, pero el riesgo general es bajo R. Farrell, Y. Ang, P. Kileen, D. O'Briain, D. Kelleher, P. Keeling y D. Weir. 2000 oct;47(4):514-9.
7. Charles N. Bernestein, MD. FRCP Active Idiopathic Ulcerative Colitis in a Patient with ongoing HIVrelated immunodepression. The Am of Gastroenterol 1991; 86: 907-9.

8. Riesgo de linfoma y tratamiento con azatioprina y 6-mercaptopurina en la enfermedad inflamatoria intestinal Elena Ricart. Servicio de Gastroenterología. Hospital Clínico de Barcelona, 2012, página 75.

9. James SP. Remission of Crohn's disease after human immunodeficiency virus infection. Gastroenterology 1988; 95: 1667-9.

10. Oncología clínica 3ra edición. Martin D. Abeloff MD., John E. Armitage, Michael B. Kastan, Gillies McKenna, 2005 Elsevier España S.A.

11. Álvaro Pizarro, Hernán García, Ana Riquelme, Javiera Carmona y Claudia Cortés, Linfomas asociados a infección por VIH en pacientes del Hospital Clínico San Borja Arriarán/ Fundación Arriarán 2001-2008. Rev. Chilena Infectol 2013; 30 (1): 23-30. 